\title{
Convection enhanced delivery for treating brain tumors and selected neurological disorders: symposium review
}

\author{
Michael A. Vogelbaum
}

Received: 20 November 2006/ Accepted: 22 November 2006/Published online: 4 January 2007

(C) Springer Science+Business Media B.V. 2006

\begin{abstract}
In 2003 the Cleveland Clinic Brain Tumor Institute sponsored a symposium to mark the progress being made in what was then a new approach to treating brain tumors-convection enhanced delivery (CED) [Vogelbaum MA (2005) J NeuroOncol 73(1):57-69]. A second symposium was held in February, 2006, to review new accomplishments and identify promising avenues of research in this evolving but still novel therapy. Among the general subjects covered by a host of international experts in their respective fields were advances in CED technology, new clinical applications of the technology, advances in CED-related imaging procedures, reviews of current or proposed trials, new drugs and the status of projects moving from lab to clinical practice. Specific subjects included the design of new catheters, the development of mathematic models for planning, novel therapeutics for CED treatment of stroke, spinal cord degenerative disease and epilepsy, liposome-based agents administered via $\mathrm{CED}$, ultra-sound driven CED, monitoring the in vivo effects of intratumoral paclitaxel and other topics. Each speaker's presentation has been abstracted along with relevant references.
\end{abstract}

Keywords Blood-brain barrier - Brain tumor . Convection enhanced delivery · Gliomas · Targeted therapy

\footnotetext{
M. A. Vogelbaum $(\bowtie)$

Brain Tumor Institute, Cleveland Clinic Foundation, 9500 Euclid Ave, Cleveland, OH 44195, USA

e-mail:vogelbm@neus.ccf.org
}

\section{Introduction}

Three years ago, in 2003, the Cleveland Clinic Brain Tumor Institute convened a symposium to describe the state of what was then the relatively new concept of convection enhanced delivery (CED) [1]. We convened a second symposium on CED in February, 2006, to mark three years of progress and chart a path into the future. The technology, although still in its infancy, retains its promise. The international panel of investigators who attended this year's meeting in Cleveland discussed new technologies, refinements of existing technologies, the development of new mathematic models that will allow therapies to be designed to meet specific clinical presentations in specific patients, refinements in imaging to allow more accurate realtime imaging of therapeutic infusions and monitor the progress of therapies, and development of new therapeutic agents amenable to CED treatment of brain tumors. In short, most every aspect of this developing field was discussed in detail. The following are summaries of the presentations.

\section{Novel catheters for CED I}

Krys Bankiewicz M.D., Ph.D. (Associate Professor of Neurological Surgery, University of California, San Francisco) began his remarks by describing a host of variables that affect the distribution of an agent within a targeted area of the brain. Among these are the character of the agent, the character of the therapy (acute or chronic), the location of the cannula, the nature of target tissue, and the physical properties of the cannula itself (i.e. its size, shape and the shape and 
number of openings at the end of the cannula or needle.) A final aspect of understanding these properties is developing imaging technology that will allow the dispersal of agents to be understood and thus better controlled.

One of the initial considerations is the diameter of the infusion cannula, which appears to have a significant affect on reflux. In this instance, reflux is not the agent moving back into the bore but the tendency of an injected agent to move back along the outer surface of the shaft between the shaft and tissue it has penetrated. It first coats the shaft and then disperses into surrounding tissue but at a different rate than it does from the tip of the cannula. The movement of an agent back along the shaft of can be rapid. There appears to be a critical size for cannula of around 26 gauge. Studies in agarose gels show that smaller cannulas perform better than larger and convect fluid at a greater rate.

A "step design cannula" consists of sequence of ever smaller shafts, each terminating at a different point along the device with the smallest at the distal end. The concept is to move an infusate from various points along the shaft at a constant rate to create a reasonably balanced dispersion pattern. One such cannula, now in gene therapy trials, is composed of four layers of 304 surgical steel fused by laser welding to a step design, ending in 23 gauge tubing. The steel cannula is lined with fused silica, which also forms the tip that extends $1 \mathrm{~cm}$ beyond the steel. The device has been dubbed by some as a "reflex resistant cannula."

There appears to be a yet to be described pumping mechanism or transport mechanism in the perivascular space that moves molecules within tissue. Magnetic resonance imaging (MRI) of liposomes injected into brain tissue shows them being distributed by this mechanism. The phenomena is being studied further.

\section{Novel catheters for CED II}

Michael Bronskill, Ph.D. (Department of Medical Biophysics, University of Toronto) asked attendees to think of CED technology not as a collection of instruments but as therapy delivery system whose configuration can be altered according to clinical circumstance. Such a system may involve varied types of cannulas, a pump, connecting tubes, flow monitors and imaging technology. The success of therapy depends on placing the agent on target and that the agent being administered assumes a volume and shape that maximizes its effect. Time is a consideration with some agents.
Successful infusion requires that the chosen cannula is inserted into the target tissue in such a manner that the agent perfusing from the instrument's multiple orifices assumes a predetermined volume and shape within a given amount of time. Clinical circumstances may dictate single infusions, multiple infusions, intermittent or continuous infusion. All these approaches require an accurate imaging technology that allows the perfused agent's shape to be monitored in real time.

Early work with multi-port catheters show they are somewhat prone to flow anomalies. It is possible that this problem can be overcome with better designs. Air entrapped in the cannula can create severe problems with uniform drug distribution and is to be avoided at all costs.

A theme that runs through a number of experiments with cannulas of different designs is that smaller bore cannulas are more likely to create good flow patterns and good pressure profiles. As cannulas get larger and/ or more complicated backflow and the shape of the volume of infusion become difficult to impossible to control.

\section{Mathematical models of CED}

Paul Morrison, Ph.D. (Division of Bioengineering and Physical Science, National Institutes of Health) proposed that the technology of CED is at a point where drug distribution in the central nervous system can be predicted with a certain degree of confidence. Among the major factors that influence CED are convective and diffusive fluxes, intracellular space (ICS) and extracellular space (ECS) partitioning, microvascular transport, extra-choroidal fluid production, metabolism, endocytosis and proelastic expansion.

$\mathrm{He}$ described mathematical means of translating physiologic-biochemical factors into equations that allow the construction of mathematical models that will produce a description of the distribution of parent metabolite concentrations in space and time in tissue. The underlying mathematical factors in these models rest on known parameters such as time-dependent conservation of mass and momentum relationships involving tissue fluids and solids and the infusate. Empirical data on the pharmacodynamics of varied agents can be incorporated to produce equations whose solutions can influence clinical decisions.

An example of this is an equation developed several years ago [2] that determines how far back along a catheter a fluid will move when dispersed under specific pressure from a catheter's tip. The pressure that 
forces the fluid from the tip of the cannula has a dual effects. It drives the fluid through the interstices in the tissue and also acts on the tissue as a whole pushing it away from the cannula to create a narrow annular space through which infusate flows toward the distal section of the instrument.

These equations that have been developed allow diffusion patterns to be pre-determined. For example, gemcitabine is a small molecule with a molecular weight of 266. A model of gemcitabine infused into gray matter at $3 \mathrm{ul} / \mathrm{min}$ through a 28 -gauge needle shows the perfused compound to assume a tear-drop shape that gradually evolves to a more spherical shape. Mathematical models of large volume infusions have to take into account the distribution of irregular geometry as the infusate is transported across white-gray boundaries. Since white matter is involved, fiberdirected flow has to be accounted for.

One of the therapeutic approaches being considered today is the infusion of a substance $\mathrm{P}$ receptor (SPR) directed neurotoxin into the spinal cord to reduce chronic neuropathic pain [3, 4]. Early work involved injecting the drug into the intrathecal space. However, a better, more physiological model involves placing a catheter into the dorsal column and using the spinal cord white matter tracts as a drug reservoir that allows the agent to move toward targeted lamina. As anticipated, the drug showed a preference to flow parallel to white fiber. This finding demonstrates that a computational model of the system has to take into account white matter anisotropy.

These and other experiments have helped the creation of formulas that govern white and gray matter diffusion, white and gray matter hydraulics and cellular porosity. The latter formula helps determine the amount of active agent actually entering the cytosol. One of the larger problems in creating mathematical models of drug delivery is defining metabolic parameters within the cytosol. If errors in estimating final responses are to creep into the formula, they are most likely to derive from the range of interacting metabolic factors affecting the concentration of the active drug.

The mathematical models developed from animal studies have helped create models of human spinal cord infusion of substance P-diphtheria toxin (SP-DT). This toxin targets neurokinin type 1 (NK1) receptor expressing neurons [4]. By plugging a range of infusate concentrations into this particular model, the authors were able to identify the optimal concentration of the therapeutic. The framework of this model allows other drugs and tracer agents to incorporated into the formula, again to determine which concentrations are likely to produce optimal results.
The work is far from complete. Study continues on white matter anisotropy. It must be remembered that these models are proceeding in three dimensions, four if time is considered to be a factor.

\section{Computational modeling of CED}

Raghu Raghavan, Ph.D. (Therataxis, Baltimore, Maryland) said that the drawbacks to early mathematic models of infusate patterns is that they were built around finite parameters. These models were informative but the clinical value of a mathematical model that accurately predicts patterns lies in its ability to be tailored to individual clinical circumstances. Dr. Raghavan and his colleagues are working with the above described NIH model of backflow to develop software that will allow for patient-specific planning of CED therapy.

It is known that the length of backflow or reflux is dependent on fluid viscosity, flow rate, the hydraulic resistance of the tissue, the outer radius of the catheter and the shear modulus or the tissues deformation by the catheter and infusion. The backflow theory is primarily a steady state theory that assumes that parameters such as pressure, flow rate and others reach an equilibrium. Dr. Raghavan challenged this assumption of equilibrium. He stipulated that the flow rate must be maintained for a significant interval before any semblance of equilibrium is reached and by the time equilibrium is reached, simple models of diffusion such as that used to predict backflow become suspect.

There are other factors to be considered in making a more robust model. Pressure forces water into the capillaries. The loss of this fluid increases the concentration of the agent. Eventually, the agent itself will move into the capillaries or will become metabolized. This also alters concentrations.

Dr. Raghavan summarized his remarks to that point in his presentation by saying that in order to compute backflow, one needs to know some of the tissue properties near the catheter. In order to compute the fluid flow in tissue, one needs to know the hydraulic conductivity and efflux. In areas of blood-brain barrier disruptions, there are interstitial variations, which further complicate the picture.

To-date, one of the factors that has not been adequately addressed is poroelasticity. In white matter, poroelasticity can be a dominating effect and there is a question as to whether the edema produced by infusion alters the tissue to a degree that additional MR scanning is required to insure the accuracy of the initial predictions. Repeated scans should be unnecessary if 
the predictions are accurate and involve all variables to include the edema.

\section{Use of advanced MRI protocols for modeling}

Martin Brady, M.D. (Therataxis, Baltimore, Maryland) described how MRI can provide much of the data needed to give mathematic models validity in a specific patient. The patient-specific factors are hydraulic conductivity, elastic moduli, pore fraction, and extracellular diffusion.

The key to acquiring data for calculating hydraulic conductivity and elastic moduli is diffusion tensor imaging (DTI) which allows the observation of molecular diffusion in tissues. A modulus of elasticity is a mathematical representation of a structure's tendency to be deformed by the force acting upon it. In the case of CED, the structures are the tissues of the brain and the force is the infused agent. Data acquired by DTI allow both the hydraulic conductivity and the elastic moduli to be calculated.

DTI provides an overall view of diffusion and information on extracellular diffusion; intracellular diffusion must calculated. Information on exchange diffusion or exchange rate does not significantly affect the overall model because the timing of the MR reduces its impact on the formula. The data that can be used to create a simple, patient-specific formula are the signals coming from the extracellular and intracellular water. Both signals are weighted with the extracellular diffusion being treated as standard Gaussian diffusion and the intracellular diffusion being treated as restricted diffusion. Treating the intracellular diffusion as restricted diffusion reduces the size of its factor and allows it to be used as a constant. DTI allows the extracellular fraction to be estimated which in turn can be used to obtain a rough estimate of the pore fraction. These are many, but not all of the variables needed to create a patient-specific mathematic model of the diffusion. Further work is underway to refine imaging and computational techniques, which will allow for the development of better, patient-specific models.

\section{CED for stroke: engineering chimeric proteins to safeguard neurons}

Although much of the discussion during the two-day symposium focused on the destruction of tumor tissue, Antonella Antignani, Ph.D. (National Institute of Neurological Disorders, NIH) offered a window into the utilization of CED to protect and preserve tissue.
The technology offers promise in preventing the neuronal destruction resulting from stroke.

Her laboratory focuses on neuronal cell death. Insults such as hypoxia or ischemia initiates apoptosis in affected cells. The laboratory is working to develop drugs that will block the apoptotic cycle and thus preserve neurons that would otherwise be destroyed. CED may well be the most effective means of getting the agent to the cells threatened by an insult, which may produce apoptosis.

Sensitivity to apoptotic stimuli is governed in large part by the expression of proteins in the BCL-2 family, which include both pro- and anti-apoptotic members. There are two proteins in the BCL-2 family that are of particular interest: BCL-2 and BCL- $\mathrm{X}_{\mathrm{L}}$. These two proteins are highly expressed during neuron development prior to birth and BCL- $\mathrm{X}_{\mathrm{L}}$, a homolog of BCL-2, is expressed both before and after birth. Study of these proteins in the rat brain shows they can block apoptosis induced by ischemia [5]. Other studies show that BCL$\mathrm{X}_{\mathrm{L}}$ can cross cellular membranes. Dr. Antignani and her colleagues have developed a chimeric protein using BCL- $\mathrm{X}_{\mathrm{L}}$ and a portion anthrax cDNA that serves as an antigen-binding domain. The resulting Lfn-BCL- $\mathrm{X}_{\mathrm{L}}$ chimera has a molecular weight of $52 \mathrm{kDa}$.

The first in vivo evidence that the chimera inhibited apoptosis came from animal studies in rat optic nerves [6]. Following injury and transection, the number of pyknotic cells decrease significantly in tissues treated with Lfn-BCL- $X_{L}$. Spinal cord injury models show that $\mathrm{BCL}-\mathrm{X}_{\mathrm{L}}$ is present in white and gray matter in undamaged cells and is also expressed in neurons and normal oligodendrocytes. The level of the protein appears to decrease following mild contusion to the spinal cord. Continuing study shows that LFN-BCL- $\mathrm{X}_{\mathrm{L}}$ protects both motoneurons and neurons in the deep dorsal horn.

Dr. Antignani said the work to-date allows the following observations and conclusions: $\mathrm{LFN}-\mathrm{BCL}-\mathrm{X}_{\mathrm{L}}$ can be transduced efficiently into neurons; a single injection of LFN-BCL- $\mathrm{X}_{\mathrm{L}}$ into a spinal cord site will diffuse into neighboring spinal cord segments; $(50 \%$ of these neighboring neurons will die following injury at an adjacent site); LFN-BCL- $\mathrm{X}_{\mathrm{L}}$ will significantly increase the number of neurons; the protein does not degrade at $24 \mathrm{~h}$ post injection; and, $\mathrm{LFN}-\mathrm{BCL}-\mathrm{X}_{\mathrm{L}}$ has the ability to significantly increase the number of neurons $24 \mathrm{~h}$ after injury. The significant sparing of dorsal horn neurons and motoneurons after LFN-BCL- $\mathrm{X}_{\mathrm{L}}$ administration suggests that the protein may be expected to improve sensory and motor recovery following spinal cord injury. Optimal outcomes may be achieved with higher dosages or more frequent injections. 
The ability of CED to obtain drug concentrations higher than those seen with systemic administration of the protein may enhance the distribution and prosurvival effects of the agent. This makes CEDmediated delivery of LFN-BCL- $\mathrm{X}_{\mathrm{L}}$ a promising approach to treating spinal cord injuries and disorders involving neuronal apoptosis.

\section{Novel therapeutics for spinal cord degenerative diseases}

Nicholas Boulis, M.D. (Center for Neurological Restoration, Cleveland Clinic) opened his discussion by observing that amyotrophic lateral sclerosis (ALS) and spinal muscular atrophy (SMA) are diseases that are tenable targets for CED therapies. The loss of motor neurons in ALS inevitably leads to death, often within five years. SMA is similar to ALS with the exception that it strikes in childhood and can evolve rapidly or slowly throughout the first 15-20 years of life. Multiple sclerosis, although not the focus of this brief discussion, is a third disease that lends itself to CED therapies. In this latter instance, CED may be a viable means of delivering factors that regulate the immune system and encourage myelinization.

Therapeutic options for ALS and SMA include replacing motor neurons and/or replacing glia, which are thought to be the source of motor neuron toxicity. Indeed, cells that pump therapeutic protein can be delivered to the spinal cord one of the limitations of such protein based therapies is their duration. Proteins are metabolized relatively quickly. The same might be said of growth factors being delivered for therapy. CED may circumvent these obstacles. It offers a means of penetrating both the parenchyma and the plasma membrane barrier to deliver proteins and engineered viruses into cells.

However, no known therapy can restore life to a dead cell; the cell must be replaced. Dr. Boulis described work elsewhere which has taken advantage of current understanding of cell differentiation to essentially grow motor neurons in vitro [7, 8]. These cells transplanted to rats with destroyed motor neurons will grow axons and dendrites and begin to synapse on muscle cells. These growth and synapse processes can occur only if neurite outgrowth inhibitor (Nogo) is suppressed. Glial cell line derived neurotrophic factor (GDNF) appears to pull the developing axons into the ventral roots. Experimental animals receiving these treatments have recovered a degree of motor function. CED may be an important technology for delivering GDNF and/or inhibitors of Nogo to the targeted repair site.
The advantages that CED offers in seeding cells to damaged areas also apply to viruses engineered to deliver therapeutic genes. The use of CED may allow concentrations of these to be maintained over time. RNA silencing allows the expression of genes and therefore the proteins they produce to be blocked. CED may have a role in delivering these new therapies.

Dr. Boulis said that outside the clinical realm, treating the spinal cord also presents an engineering challenge. The cord can tolerate a range of insults such as manipulation, the insertion of a needles and the expansion of tissue or volume as happens when a tumor grows. It cannot tolerate hemisection or transection, repeated stress or traction, constriction, or inflammation. These obstacles can be overcome with mechanics. A system has been designed that immobilizes the cannula to be mounted to the spine. The cannula, supports, delivery system and entire platform would move as a single unit to allow programmed delivery of therapeutics.

\section{Restorative biodelivery in neurodegenerative disorders}

Lars U. Wahlberg, M.D., Ph.D. (NsGene A/S, Denmark) discussed some of the therapies being described such as encapsulated cell (EC) biodelivery, a technology whose character is somewhat similar to CED systems.

The delivery system involves implanting a $60 \mathrm{~mm}$ diameter catheter-like device into targeted areas of the brain. The distal end of the device is a semi-permeable membrane that allows the diffusion of molecules with molecular weights of up to $90 \mathrm{kDa}$ to move into the surrounding tissue. Dr. Wahlberg noted the membrane can be engineered to allow molecules of up to $300 \mathrm{kDa}$ to pass through. The implant holds cells that have been genetically modified to produce therapeutic proteins. The pores in the membrane are sized allow proteins to move from the cells into the surrounding tissue and nutrients to move into the implant to feed the cells. However, the pores are too small to allow immune cells to reach the modified cells. His company is working with the Karolinska Institute to develop therapies for Alzheimer's disease and with Lund University to develop treatments for Parkinson's disease.

Alzheimer's disease is a diffuse neurodegenerative disease with cholinergic degeneration affecting cholinergic neurons in the basal forebrain, cortex and hippocampus. These particular disease locations are noted because animal models of the disease suggest 
that halting degeneration in these regions retards the symptomatic development of the disease. This cholinergic system is normally supported by nerve growth factor (NGF) but as the disease progresses, the signals carried from the nerve endings to the cell bodies is impaired. Delivering NGF locally to cell bodies restores the system and permits distal functioning.

Clinical trials now being planned will involve six patients. Three will receive bilateral EC implants into the nucleus basalis. Cortical projections from the nucleus basalis control attention. The second three patients will receive bilateral EC implants and bilateral anteriomedial implants. Anteriomedially located cholinergic neurons project to the hippocampus and control learning and memory. The initial responses will be monitored with positron emission tomography (PET) but cognitive outcomes will also be evaluated.

Glial cell line derived neurotrophic factor is a protein involved in the generation of striatal projections of dopaminergic cells [9-12]. It is being studied for its potential protective and regenerative potential in Parkinson's disease. Animal experiments have shown that GDNF can offer a degree of protection to cells in the substantia nigra that are insulted with injected neurotoxins. Significant regeneration of TH-positive nerve endings of dopaminergic cells has been observed in animal models [13]. It appears that the encapsulated GDNF can regenerate striatal neurons.

\section{In vivo imaging of $\mathrm{CED}-$ human studies}

John Sampson, M.D., Ph.D. (Duke University Medical Center) described early attempts to obtain single photon emission computed tomography (SPECT) and MRI images of CED diffusion patterns with the use of radiolabeled albumin. These images were among the first to offer some idea of convection induced movement of agents in the brain and they produced some surprises. Among the initial findings was the discovery that CED produced large and fairly homogenous distributions of infusate. However, significant portions of the brain remained uninfused. One of the surprising and perhaps disappointing findings was that in a majority of the infusions, the drug did not enter the brain. Of 16 infusions imaged in eight patients, seven $(44 \%)$ of the infusions showed a partial subarachnoid leak. Two $(12 \%)$ of the infusions showed a partial ventricular leak. There was a partial pooling of the agent in a resection of the necrotic area in four $(26 \%)$ of the infusions. Only three (19\%) of the infusions could be considered a success.
The images showed that whenever the pia was perforated by the tip of the catheter, there was no infusion into the parenchyma. Catheter tips that approached the pia, but did not penetrate it, provided good distribution. Dr. Sampson said that it appears that the pia is a significant barrier and that optimal distribution may be obtained by placing the catheter tip close to the pia without penetrating it.

Unlike the pia, the ependyma or resection cavities provided no barrier to infused material. Imaging also showed that when catheters crossed the deep sulci, infused material tended to follow the pre-resection catheter tract as if it were the path of least resistance.

Experience with intra-tumoral infusions in this series was limited but suggested that such infusions were not particularly homogeneous. The material tended to either pool in the necrotic center of the tumor or find a pathway of least resistance through the enhancing portion of the tumor while leaving the rest of the tumor uncovered. It was initially hoped the natural egress of fluid from the tumor would push the drug from the tumor but that did not happen.

Dr. Sampson and his colleagues also evaluated CED related changes in T2 signal. In 6 of 18 catheter placements they found no change in $\mathrm{T} 2$ signals with infusion. This allowed the identification of infusions that failed to provide intraparenchymal drug distribution on SPECT. Five catheters placed into areas of low pre-existing T2 signal showed signal changes during infusion that were highly correlated with the volume and geometry of ${ }^{123}$ I-HSA distribution. In seven catheters placed in regions of pre-existing high $\mathrm{T} 2$ signal abnormalities, $\mathrm{T} 2$ changes were noted but quantitative correlations could not be established. One of the lessons of this study was that if there is no T2 signal change after infusion, there is likely no infusion into the brain.

Dr. Sampson discussed advances being made in the software components of imaging systems, in particular programs developed by BrainLAB and Therataxis LLC for use with MRI and SPECT. The program's algorithm provides two useful pieces of information. It will determine if a sulcus is present and will offer predictions as to geometric volume distribution. In recent trials, the sensitivity of the sulcus detection component was $71 \%$ with a much lower specificity. The average volume match was 60 $70 \%$. The mean in-plane deviation was less than a centimeter. The defined clinical utility of the program was $86 \%$ allowing Dr. Samson to observe that even with its current limitations, the system has clinical utility. 
Dr. Sampson concluded his presentation with a discussion of a study of immunological responses in the presence of tumors. To answer the question as to whether tumors were responding directly to the toxicity of an agent or whether the agent was promoting a secondary immunologic response against the tumor, the investigators obtained mice with EGFRvIII overexpressing GBM tumors and treated them with either a toxin (MR1-1) specific to the EGF receptor or PBS. The tumors injected with the toxin disappeared. Some 65 days after the treatment, the mice were again challenged with EGFRvIII-positive tumors. The mice appeared to be protected from this secondary tumor challenge as there was no tumor take. Subsequent investigation revealed that the efficacy of the toxin was significantly reduced in the absence of CD4+ and CD8+ T-cells and NK cells. Dr. Samson concluded that although the work was preliminary, it appeared that the toxins lose a significant amount of their efficacy in the absence of an immune response.

\section{In vivo imaging of CED}

Russell R. Lonser, M.D. (National Institute of Neurological Disorders and Stroke, National Institute of Health) said that the ability to use imaging technology to monitor infusions is one of the emerging advantages of CED therapy. Dr. Lonser described his work with two primary groups of surrogate tracers for imaging, those that are used with MRI and those used with computer tomography (CT). He observed that one of the primary differences between MRI and CT is that CT imaging can determine concentrations based on Hounsfield units whereas the ability to use signal on MRI to determine concentration is still being investigated.

The first group of MR agents discussed were compounds with high molecular weights. These were employed because their size approximated that of many of the immunotoxins and other proteins being infused into tumors [14]. One of these, a $76 \mathrm{kD}$ gadoliniumbound albumin molecule, was shown to provide clear well-defined images in animals. Continuing study of the compound shows that it has a high correlation of distribution with the therapeutic agent $\mathrm{I}^{125}$-labeled interleukin 13 bound to Pseudomonas exotoxin (IL13-PE) in rats and primates [15]. Autoradiograms of the gadolinium tracer superimposed on autoradiograms of $\mathrm{I}^{125}$-IL13-PE treated brains revealed a $95 \%$ correlation in volume.

Gadolinium-diethylenetriaminepentaacetic acid (Gd-DTPA) is a readily available small molecule tra- cer that produces clear images. The images produced by Gd-DTPA correlated to within $13.5 \%$ of images produced by tritiated gemcitabine. This $13.5 \%$ difference amounts to about a $0.6 \mathrm{~mm}$ difference in the diameters of the images, a difference barely apparent to the eye on MR imaging. He also noted that these small molecule tracers were inaccurate at tracing larger molecule therapeutic infusions.

Iopanoic acid covalently bound to albumin creates a $70 \mathrm{kD}$ molecule frequently used in CT imaging. It provides a clear picture with good correlation of intensity in Hounsfield units. Dr. Lonser described a study which examined the safety and utility of an iodine-based low-molecular weight surrogate tracers for CT performed during CED in four primates [16]. The distribution of the tracer was determined by realtime postinfusion CT as well as quantitative autoradiography of small molecules (sucrose-MW 359 D) or large molecules (dextran-MW $70 \mathrm{kD}$ ). The distribution of the infusate was identifiable for up to $72 \mathrm{~h}$ with no evidence of toxicity. The authors concluded that real-time in vivo CT scanning of CED iopamidol appeared to be safe, feasible and suitable for monitoring CED delivery of drugs at low infusion volumes.

Dr. Lonser presented a brief case study of a child with Gaucher's disease to illustrate how animal studies translate to clinical applications. The study was conducted to determine whether glucocerebrosidase could be delivered successfully by CED and reverse the child's neurological decline. The child was infused twice with a concentration of $1 \mathrm{mM}$ of glucocerebrosidase mixed with gadolinium DTPA. The first infusion was in the frontal lobe but was conducted without the tracer. The investigators found that they were unable to track the infusate with either T2 or FLAIR imaging. The right pontine region was subsequently infused with glucocerebrosidase and gadolinium DPTA through a silicate cannula. The team was able to visualize the infusion in the right posterior pontine region during $4 \mathrm{~h}$ of the $6 \mathrm{~h}$ procedure. During this infusion, it became apparent that some of the infusate was lost into the CSF space as a pial barrier had been violated. Hence, the real-time imaging provided valuable information about catheter position and infusate flow.

Dr. Lonser concluded surrogate markers provide well-defined images on either MRI or CT. Small molecule tracers have limitations in terms of their ability to track the infusion of an infusate that contains macromolecules. Nevertheless, the tracers used to date appear to be safe and they appear to have no affect on the activity of therapeutic agents. 


\section{Monitoring the effects of intratumoral paclitaxel (Taxol) with MRI and FET-PET}

There are an increasing number of models of drug infusion, approaches to imaging the infusion and approaches to imaging drug metabolism. Roland $\mathrm{H}$. Goldbrunner, M.D. (Department of Neurosurgery, University of Munich, Germany) described a collaborative phase I/II study of taxol infusion by CED in nine patients with recurrent glioblastoma multiforme. He noted that earlier studies of taxol in vitro showed that it destroyed targeted cells but in vivo studies showed it to have little to no efficacy owing to its inability to cross the blood-brain barrier [17-23]. The trial he described involved the implantation of two ventricular catheters into the geometric center of the tumors as determined by MRI and later by fluorodeoxyglucosepositron emission tomography (FDG-PET). Dr. Goldbrunner noted that FDG-PET data allowed better catheter placement. He proposed that imaging with $O$ (2-[18F]fluoroethyl)-L-tyrosine-PET (FET-PET) would provide a better method to follow tumor growth than with FDG-PET or MRI. Patients were infused with a concentration of $0.25 \mathrm{mg} / \mathrm{ml}$ taxol at a rate of $1.8 \mathrm{mg} /$ day for five days. MRI and FET-PET was performed at baseline. Diffusion-weighted MRI was performed during convection on days 3 and 6 . PET was performed every 3 months there after.

All nine patients showed a response with a median progression-free survival of 39 weeks and overall survival of 58 weeks. The one-year progression-free survival was $33.3 \%$ and one-year overall survival was $55.6 \%$. The mean standardized uptake value (SUV) in these patients was 3.2. After CED this was reduced by $17 \%$ to a value of 2.6 with the observation that the SUV around the catheter tip was 2.0. When the tumors recurred, there was a dramatic $46 \%$ increase in FET uptake. In at least one patient the FET uptake was larger than the area of MRI contrast enhancement. Dr. Goldrunner summarized by saying that intratumoral infusion of Taxol is effective but carries a significant risk of side effects. There was good correlation between images and FET uptake. It appears that FETPET is more sensitive than MRI in estimating tumor extent and is significantly more specific in diagnosing recurrences.

\section{The value of intraoperative $\mathrm{CT}$}

Manfred Westphal, M.D. (University Hospital, Eppendorf, Germany) described recent advances in CT hardware and software, which may aid in catheter placement. He said that frame-based CT offers optimal control although the setup might be considered to be laborious. The University Hospital in Eppendorf has a dedicated CT that is linked to a mobile operating table. The CT apparatus is mounted on fixed rails allowing it to be moved when surgeons need access to the patient and returned to its exact location for scanning. Westphal described this prototype technology as being extremely accurate, a quality that is a welcome virtue in conducting CED procedures. The technology allowed for extremely accurate placement of the catheters and real-time verification of accurate placement.

\section{Liposome-based CED therapeutics}

Krys Bankiewicz, M.D., Ph.D. (Associate Professor of Neurological Surgery, University of California, San Francisco) described the potential of liposomes as vehicles for delivering a wide variety of agents to target regions and cells, calling them "universal delivery vehicles." The categories of agents liposomes can carry include drugs, dyes, probes, imaging agents and genetic material such as cDNA, siRNA, and viral vectors. Monoclonal antibody structures can be incorporated into liposomal membranes to allow the structure to bind to specific cell receptors. The most frequently used liposomes are around $100 \mathrm{~nm}$ but they can be as small as $40 \mathrm{~nm}$. Smaller liposomes limit the amount of specific agents that can be carried to a target. The structures are for the most part neutral in the biological environment and although they are biodegradable, they have a significant half-life. Pegylated liposomal doxorubicin has a half-life of up to $50 \mathrm{~h}$ as compared to $10 \mathrm{~min}$ for the naked agent $[24,25]$. Initially the cationic liposomes did not convect well, a problem that he related to the cationic charge, but this problem was overcome by peglyating [attaching polyethylene glycol (PEG)] to the liposomes. Such peglyated structures are sometimes called 'stealth' liposomes for their ability to move in a biologic environment without engendering a response. The peglyated liposomes are readily convected and unlike other vectors they have the ability to reach targeted tissue with close to $100 \%$ efficiency. Dr. Bankiewicz showed photos of a liposomal-enclosed marker reaching nearly every cell in the U87MG tumor model. The liposome-delivered molecule did not discriminate between neurons, astrocytes or tumor cells said Dr. Bankiewicz. Another virtue liposomes possess as vectors is that they also appear to diffuse well.

Dr. Bankiewicz turned the discussion to specific agents, notably the topoisomerase inhibitor irinotecan (CPT11) and doxorubicin. These drugs can be effi- 
ciently encapsulated in liposomes at high concentrations, up to $10^{5}$ molecules per liposome. Animal studies show that when injected into peripheral circulation, the free drug is cleared within $6 \mathrm{~h}$ but in liposomes it remains stable for up to $48 \mathrm{~h}$ or longer and can be detected for up to 50 days after a single injection. Encapsulation also affects toxicity. A $20 \mathrm{mg} / \mathrm{ml}$ dose of free drug convected into a rat brain is seen to be highly toxic whereas liposomal doses of up to $80 \mathrm{mg} / \mathrm{ml}$ evidence no toxicity.

Experiments with U87MG tumors in rats show significantly increased survival. All animals receiving free CPT11 at $3 \mathrm{mg} / \mathrm{ml}$ died within 35 days. More than half the animals given liposomal CPT11 at $80 \mathrm{mg} / \mathrm{ml}$ remained alive for more than 100 days. Similar experiments with free topotecan versus liposomal topotecan showed that animals receiving the free form perished within 30 days following tumor implantation where as more than $80 \%$ of those receiving the liposomal form remained alive at 100 days. It was observed by an audience member that although liposomes have traditionally been considered to be fragile or unstable structures that would be expected to breakdown under the pressure of convection, the toxicology of free versus liposomal studies suggest this is not the case. Responding to another query Dr. Bankiewicz said that although CPT11 underwent decarboxylation to create its active metabolite, SN38, the process may not be necessary because robust destruction of cancer cells is seen with the non-decarbolized agent suggesting that CPT11 may be an active molecule in itself. He added that a number of experiment show that CPT11 does not undergo conversion to $\mathrm{SN} 38$ in tumors yet remains active.

\section{Spontaneous canine gliomas: a model system for CED}

Peter Dickinson, D.V.M. (University of California Davis Veterinary Teaching Hospital) described the potential that dogs with spontaneous brain tumors may have for conducting investigations into optimizing CED. Such tumors appear in dogs infrequently, but more frequently than in humans, about 14.5 per 100,000 in the animals as compared to $4-9$ per 100,000 in humans.

Distribution of histologies is slightly different in canines than in humans but all types are seen. Tumor histologies observed in dogs include astrocytomas, oligodendrogliomas, ependymomas and choroid plexus papillomas. About half of the canine tumors coming to Dr. Dickinson's clinic are meningiomas. Overall, only about $20 \%$ of tumors are glioblastoma multiforme. More commonly, grade 3 astrocytomas are observed. The majority of gliomas appear in brachycelphalic (squashed nosed) dogs such as boxers, bulldogs and Boston terriers. About $90 \%$ of the oligodendrogliomas present in Boxers.

The histologic features of canine brain tumors are similar to those seen in humans. These tumors range from grade 2 to grade 4 and include classic glioblastomas containing serpentine necrosis, pseudopalisading, microvascular proliferation and other clinical features. These tumors express many other molecular alterations associated with human tumors to including overexpression of VEGF, VEGFR-1, VEGFR-2, EGFR1 PDGFR $\alpha$, PDGF $\alpha / \beta$, PPDGER $\beta$, and IL13R2 $\alpha$. It has not yet been determined whether the homologue to $1 \mathrm{p} / 19 \mathrm{q}$ deletions is observed in canine oligodendrogliomas.

Although all the cited factors seem to make dogs ideal candidates as animals for CED trials, it is important to recognize that these glioma-bearing dogs are pets whose owners may have the same difficulty in consenting to experimental procedures and treatments as they would for other (human) family members.

Dr. Dickinson described the development of a program to study tumors, therapies and technologies in these animals and his group has developed an MRcompatible frame for imaging and drug delivery. Dogs, like primates, can be active so catheters must be well anchored. The team at the hospital has had success with UV curing dental acrylic, which is bound to brass screws in the skull. He presented investigations with CED infused liposomal CPT11/gadolinium. The catheters were left in the dogs for three weeks before the animals were euthanized. Histology showed very little if any toxicity in the region of the liposomal infusion. There was a very mild perivascular response that appeared to involve macrophages. Dr. Dickinson wondered aloud if the changes in post-infusion enhancement seen in humans might be perivascular reactions.

A case study was presented of a nine-year-old spayed Jack Russell terrier diagnosed with a grade 3 astrocytoma. The readily observable glial filaments were strongly GFAP-positive. CED was used to infuse liposomal CPT11/Gd at a rate of about $3 \mathrm{ml} / \mathrm{min}$. As in some of the human studies presented in this symposium, the infused compound reached a finite volume of distribution because the leading edge reached an ependymal surface, the temporal horn of the animal's left ventricle. Leakage developed and the volume of distribution became static despite continuing infusion of drug. 
Dr. Dickinson concluded his presentation saying that CED of CP11 is safe in dogs, placing infusions directly into tumors via CED is a realistic goal, realtime monitoring of the process allows rapid identification of evolving problems, and that canine models are likely to provide useful in vivo data for the development of CED therapies.

\section{CED of agents to treat medically intractable epilepsy}

John Heiss, MD, (National Institute of Neurological Disorders and Stroke, National Institute of Health) described investigations with CED in treating epilepsy. The hippocampus is the primary site of surgically remediable, medically intractable epilepsy and this would be the presumed target of CED based therapies. There are two approaches to treating epilepsy with CED, neurophysiologic and pharmacologic. The former approach would involve delivering a selective neurotoxin to destroy excitatory outflow from the dentate gyrus of the hippocampus. The pharmacologic approach would infuse a reversible inhibitory neurotransmitter into the ECS of the epileptic focus where it can act on the post-synaptic membrane to suppress hippocampal outflow.

The standard treatment of mesial temporal sclerosis, the most common epileptic syndrome in adults, is to remove on the non-dominant side about $51 / 5$ to $6 \mathrm{~cm}$ of the tip of the anterior temporal lobe. At one year, $64 \%$ of the patients undergoing this procedure are free of disabling seizures but only about $38 \%$ were free of all seizures including auras [26]. Some $10-15 \%$ saw no improvement. He noted that in one of the few controlled trials comparing surgery to medical treatment, one of the patients in the medical treatment arm died showing that epilepsy is not a benign disease.

There is an effort by the National Institute of Neurological Disorders and Stroke to develop CED as a treatment approach reasoning that it may improve outcomes and reduce side effects. Muscimol, a GABAA receptor agonist, was chosen because GABA is the principle inhibitory neurotransmitter in mesial temporal sclerosis. Models show a loss of GABA-ergic neurons in the granule layer of the hippocampus. Dr. Heiss and his team chose to uses monkeys as a large animal model for a preclinical study looking at safety and distribution of muscimol delivered by CED.

The FDA required that the study design include a treatment cross-over. Catheters were implanted in ten animals with four of them acting as controls and receiving no infusions. Three animals received muscimol and three received Elliot's B solution as a vehicle. Muscimol and the vehicle were infused at a rate of $84 \mu \mathrm{l} / \mathrm{h}$ for a little over $13 \mathrm{~h}$. On day 11 , the muscimol animals were switched to Elliot's B solution and those receiving Elliot's B were given Elliot's B plus radiolabled muscimol to allow for autoradiography at the end of the experiment.

MRI and radiography showed the infused fluids stayed within the hippocampus. Concentrations of infusate ranged from $0.7 \%$ to $7 \%$ throughout the targeted temporal lobe with no muscimol detected in the contralateral temporal lobe.

At $24 \mathrm{~h}$ post infusion the fluid appeared to be completely reabsorbed. Animals receiving muscimol at $14 \mu \mathrm{g} / \mathrm{ml}$ appeared normal. The muscimol reduced EEG frequency. Those receiving the agent at $28.5 \mu \mathrm{g} /$ $\mathrm{ml}$ seemed to be calmed and those receiving $57 \mu \mathrm{g} / \mathrm{ml}$ became lethargic. Histologic examination of the region infused revealed some inflammatory cells around the cannula site but the tissue otherwise appeared to be completely normal. This study indicated that the infusion of muscimol into the hippocampus by CED is both feasible and safe. Further development of this antiseizure treatment approach is ongoing.

\section{Ultrasound mediated interstitial drug delivery}

Andrew Kanner, M.D. (Tel Aviv Sourasky Medical Center, Israel) and his colleagues have been working on a new delivery system that utilizes ultrasound as the primary force driving drug dispersion from specially designed catheters. The device consists of a primary catheter inside a $1.2 \mathrm{~mm}$ diameter tube. The soundwave generated at the distal end of the device are translated to a low amplitude mechanical force at the tip of the device driving nano-particles through dispersion windows at the tip. A feeding system near the proximal end of the catheter allows agents to enter the system at an even flow.

The initial trials of the device were conducted in rats with iron oxide nano-particles, which are $15-20 \mathrm{~nm}$ in size when dry and 50-70 nm wet. The particles were coated with dextran to simulate a drug-coated particles. One of the therapeutic approaches under consideration is to deliver nano-particles coated with slow release agents. The particles in a saline solution were driven from the dispersal window at the distal end of the catheter (the tip is sealed) by a $1-2 \mathrm{~W}$ power source delivering a pulse duration between $1 \mathrm{~s}$ and $2 \mathrm{~s}$ with amplitudes of $100-150 \mu \mathrm{m}$ at the tip. Infusion times ranged from $1 \mathrm{~mm}$ to $2 \mathrm{~min}$, which produced a 
homogenous distribution of the particles covering most of the frontal lobe of a rat.

A series of experiments with gadolinium DTPA, as the agent, showed no significant dispersion. Dr. Kanner explained that the gadolinium solution behaved like a fluid whereas the nano-particles had completely different properties and were not necessarily being pushed through brain tissue like a fluid.

Histological examination of the tissue showed localized trauma at the insertion site and along the track of the probe but no signs of damage at the periphery. Experiments in cow brains showed the technology was able to disperse nano-particles over distances up to $3 \mathrm{~cm}$ in as little as $2 \mathrm{~min}$. The resonance frequency of the brain tissue was found to be very narrow and distribution halted when the frequency drifted. A new probe was designed to have a feedback mechanism that detects resonance drift and automatically corrects for it. Initial pharmacological data derived from particles coated with Adriamycin and others coated with Taxol are being analyzed.

Responding to queries from the audience he said he did not think the boundaries of cells and tissues would be as great an issue with nano-particles as it appeared to be with fluids in CED although that has yet to be shown in tumor models. The particles his group have used are small enough to pass through tissues. MRI scans to date have shown no necrosis or edema in or near the treatment area.

\section{Clinical and basic advances in CED: Optimizing convection for better clinical outcomes}

Zvi Ram, M.D. (Tel Aviv Medical Center, Israel) opened his remarks by identifying some of the challenges that needed to be overcome in order to optimize the delivery agents that may someday produce better clinical outcomes that current standards of therapy.

Anatomy plays a role in determining diffusion. Diffusion tensor images can be fused with intraoperative images and used as a tool to place catheters but white matter, anatomic structures and barriers such as scar tissue, gliosis, sulci and others can influence diffusion patterns as was evidenced by the lengthy discussion of the problems of reflux or backflow that were discussed early in the symposium. The toxicity of agents is a two-pronged problem because it is difficult at this point to control either the effect of the drugs being used or their exact placement he said.

Imaging the convection offers a means of assessing efficacy and safety. He described work being done by Yael Mardor, Ph.D., of the Chiam Sheba Medical
Center, Tel Hashomer, Israel. Dr. Mardor has shown that diffusion can be effectively imaged if gadolinium diluted to a 1 in 70 concentration is added to the infusate. Imaging response to treatment by a variety of agents is possible with diffusion weighted MRI. The agents include taxol and platinum-based compounds whose response can be imaged within hours to immunological agents whose response may not appear for several days.

Choosing the appropriate drugs for CED can be problematic. The requirements are that the drug under consideration must be non-toxic to normal tissue and stable in situ. Individual agents have individual characteristics. Viscosity is a consideration. There is a linear correlation between viscosity, volume of convection, and the incidence of backflow he said. The size and molecular weight of drugs can affect convection and diffusion. He surmised that particles up to $100 \mathrm{~nm}$ can probably be convected. This is a size far larger than thought possible only a few years ago. Dr. Ram pointed out all CED work is progressing under the assumption that better distribution is associated with increased efficacy but that the hypothesis had yet to be proven.

Dr. Ram advised the audience that CED is a regional treatment and as such, it is limited in its scope and goals. As his last clinical point, he noted that intratumoral CED procedures may be significantly different from peritumoral approaches. Each procedure must be tailored according to the mode of convection, the specific infusate being used, the flow rate, the character of the catheters employed and the clinical characteristics of the patient being treated.

\section{Future directions}

The pace of development of the field of CED depends heavily on the outcome of the first phase III trial to complete patient accrual (NeoPharm Inc.'s PRECISE trial). This trial, which was closed in December, 2005, randomized patients, with recurrent GBM treated with a gross-total resection of the recurrent enhancing disease, to treatment with cintredekin besudotox $(\mathrm{CB})$ via $\mathrm{CED}$ or with Gliadel ${ }^{\circledR}$ chemotherapy wafers implanted at the time of surgery. The results of this trial are expected to be available in early 2007. If this trial shows a sufficient survival benefit following treatment with $\mathrm{CB}$, it is expected that further development of this drug (and other related drugs) will proceed at an accelerated pace. On the other hand, failure of this trial to show 
benefit of the experimental regimen will likely result in a much slower pace of progress due to the significant cost of clinical trials that involve the implantation of intracerebral catheters for CED. In that case, further clinical advances would be more likely to arise from small, single center trials or from non-glioma applications of CED. The Third International Symposium on Convection Enhanced Delivery will be held in Cleveland in May, 2007, at which time the results of this trial and other updates from the field of CED will be presented and discussed.

Acknowledgements The author would like to thank Martha Tobin for her invaluable assistance organizing the 2006 International Symposium on Convection Enhanced Delivery. Christine Moore and Mac Overmyer provided editorial assistance for this report. The symposium was funded by an unrestricted educational grant from BrainLAB AG. The author is a consultant to and scientific advisory board member for NeoPharm, Inc.

\section{References}

1. Vogelbaum MA (2005) Convection enhanced delivery for the treatment of malignant gliomas: symposium review. J NeuroOncol 73(1):57-69

2. Bobo RH, Laske DW, Akbasak A, Morrison PF, Dedrick RL, Oldfield EH (1994) Convection-enhanced delivery of macromolecules in the brain. Proc Natl Acad Sci USA 91(6):2076-2080

3. Sarntinoranont M, Banerjee RK, Lonser RR, Morrison PF (2003) A computational model of direct interstitial infusion of macromolecules into the spinal cord. Ann Biomed Eng 31:448-461

4. Sarntinoranont M, Iadarola MJ, Morrison PF (2003) A kinetic analysis of substance $\mathrm{P}$ trafficking. $\mathrm{J}$ Pharm Sci 92:232-243

5. Liu XH, Castelli JC, Youle RJ (1999) Receptor-mediated uptake of an extracellular Bcl-xL fusion protein inhibits apoptosis. Proc Natl Acad Sci USA 96:9563-9567

6. Liu XH, Collier RJ, Youle RJ (2001) Inihibition of axotomyinduced neuronal apoptosis by extracellular delivery of a Bcl-XL fusion protein. J Biol Chem 49:46326-46332

7. Harper JM, Krishnan C, Darman JS, Deshpande DM, Peck S, Shats I, Backovic S, Rothstein JD, Kerr DA (2004) Axonal growth of embryonic stem cell-derived motoneurons in vitro and in motoneuron-injured adult rats. Proc Natl Acad Sci USA 101:7123-7128

8. Kerr DA, Llado J, Shamblott MJ, Maragakis NJ, Irani DN, Crawford TO, Krishnan C, Dike S, Gearhart JD, Rothstein JD (2003) Human embryonic germ cell derivatives facilitate motor recovery of rats with diffuse motor neuron injury. $\mathbf{J}$ Neurosci 23:5131-5140

9. Georgievska B, Jakobsson J, Persson E, Ericson C, Kirik D, Lundberg C (2004) Regulated delivery of glial cell linederived neurotrophic factor into rat striatum, using a tetracycline-dependent lentiviral vector. Hum Gene Ther 15:934-944

10. Georgievska B, Carlsson T, Lacar B, Winkler C, Kirik D (2004) Dissociation between short-term increased graft survival and long-term functional improvements in Parkinso- nian rats overexpressing glial cell line-derived neurotrophic factor. Eur J Neurosci 20:3121-3130

11. Georgievska B, Kirik D, Rosenblad C, Lundberg C, Bjorklund A (2002) Neuroprotection in the rat Parkinson model by intrastriatal GDNF gene transfer using a lentiviral vector. Neuroreport 13:75-82

12. Kirik D, Georgievska B, Rosenblad C, Bjorklund A (2001) Delayed infusion of GDNF promotes recovery of motor function in the partial lesion model of Parkinson's disease. Eur J Neurosci 13:1589-1599

13. Sun ZH, Lai YL, Li P, Zuo HC, Xie ZP (2004) GDNF augments survival and differentiation of $\mathrm{TH}$-positive neurons in neural progenitor cells. Cell Biol Int 28(4):323-325

14. Nguyen TT, Pannu YS, Sung C, Dedrick RL, Walbridge S, Brechbiel MW, Garmestani K, Beitzel M, Yordanov AT, Oldfield EH (2003) Convective distribution of macromolecules in the primate brain demonstrated using computerized tomography and magnetic resonance imaging. J Neurosurg 98:584-590

15. Gregory JA, Murad GJA, Walbridge S, Morrison PF, Garmestani K, Degen JW, Brechbiel MW, Oldfield EH, Lonser RR (2006) Real-time, image-guided, convection-enhanced delivery of interleukin 13 bound to Pseudomonas exotoxin. Clin Cancer Res 12:3145-3151

16. Croteau D, Walbridge S, Morrison PF, Butman JA, Vortmeyer AO, Johnson D, Oldfield EH, Lonser RR (2005) Real-time in vivo imaging of the convective distribution of a low-molecular-weight tracer. J Neurosurg 102:90-97

17. Walter KA, Cahan MA, Gur A, Tyler B, Hilton J, Colvin OM, Burger PC, Domb A, Brem H (1994) Interstitial taxol delivered from a biodegradable polymer implant against experimental malignant glioma. Cancer Res 54:2207-2212

18. Cahan MA, Walter KA, Colvin OM, Brem H (1994) Cytotoxicity of taxol in vitro against human and rat malignant brain tumors. Cancer Chemother Pharmacol 33:441-444

19. Silbergeld DL, Chicoine MR, Madsen CL (1995) In vitro assessment of Taxol for human glioblastoma: chemosensitivity and cellular locomotion. Anticancer Drugs 6(2):270276

20. Terzis AJ, Thorsen F, Heese O, Visted T, Bjerkvig R, Dahl O, Arnold H, Gundersen G (1997) Proliferation, migration and invasion of human glioma cells exposed to paclitaxel (Taxol) in vitro. Br J Cancer 75:1744-1752

21. Fetell MR, Grossman SA, Fisher JD, Erlanger B, Rowinsky E, Stockel J, Piantadosi S (1997) Preirradiation paclitaxel in glioblastoma multiforme: efficacy, pharmacology, and drug interactions. New Approaches to Brain Tumor Therapy Central Nervous System Consortium. J Clin Oncol 15:31213128

22. Chang SM, Kuhn JG, Robins HI, Schold SC Jr, Spence AM, Berger MS, Mehta M, Pollack IF, Rankin C, Prados MD (2001) A Phase II study of paclitaxel in patients with recurrent malignant glioma using different doses depending upon the concomitant use of anticonvulsants: a North American Brain Tumor Consortium report. Cancer 91:417-422

23. Hurwitz CA, Strauss LC, Kepner J, Kretschmar C, Harris MB, Friedman H, Kun L, Kadota R (2001) Paclitaxel for the treatment of progressive or recurrent childhood brain tumors: a pediatric oncology phase II study. J Pediatr Hematol Oncol 23:277-281

24. Coukell AJ, Spencer CM (1997) Polyethylene glycol-liposomal doxorubicin: a review of its pharmacodynamic and pharmacokinetic properties, and therapeutic efficacy in the management of AIDS-related Kaposi's sarcoma. Drugs 53(3):520-538 
25. Gill PS, Wernz J, Scadden DT, Cohen P, Mukwaya GM, von Roenn JH, Jacobs M, Kempin S, Silverberg I, Gonzales G, Rarick MU, Myers AM, Shepherd F, Sawka C, Pike MC, Ross MC (1996) Randomized phase III trial of liposomal daunorubicin versus doxorubicin, bleomycin, and vincristine in AIDS-related Kaposi's sarcoma. J Clin Oncol 14(8):23532364
26. Wiebe S, Blume WT, Girvin JP, Eliasziw M (2001) Effectiveness and Efficiency of Surgery for Temporal Lobe Epilepsy Study Group. A randomized, controlled trial of surgery for temporal-lobe epilepsy. N Engl J Med 345:311-318 